\title{
Secondary Radiation Field Effects for the CEM Spectra
}

\author{
K. Ruebenbauer ${ }^{a, *}$, A. BŁAChowski ${ }^{a}$ And J. Żukrowski ${ }^{b}$ \\ ${ }^{a}$ Mössbauer Spectroscopy Division, Institute of Physics, Pedagogical University \\ Podchorążych 2, PL-30-084 Kraków, Poland \\ ${ }^{b}$ Faculty of Physics and Applied Computer Science, AGH University of Science and Technology \\ al. Mickiewicza 21, PL-30-059 Kraków, Poland
}

(Received July 23, 2009; in final form March 9, 2010)

\begin{abstract}
The secondary resonant radiation field and resonant absorption thickness effects on the Conversion Electron Mössbauer Spectroscopy spectra are analyzed for highly enriched resonant targets. It is shown that secondary field effect is important for the thick $\alpha$-Fe foil enriched in the resonant isotope. Even for the polycrystalline sample traces of the coherent resonant field have been detected as the distortion of lines. Secondary field is discussed in detail. Suitable approximations to treat spectra originating from targets with significant resonant thickness developing secondary field composed of the incoherent and coherent parts are introduced. Finally, the formalism is applied to the high quality spectrum recorded for the enriched iron foil and correlation between contribution due to the secondary field and experimental line shape is investigated.
\end{abstract}

PACS numbers: 76.80. $+\mathrm{y}, 82.80 . \mathrm{Ej}, 23.20 . \mathrm{Nx}, 42.40 . \mathrm{Kw}$

\section{Introduction}

Usually Mössbauer spectra recorded by means of the secondary radiation emerging from the resonant target illuminated by the resonant beam are analyzed in the thin target (Lorentzian) approximation. Such approach is particularly justified while using back scattered secondary electrons to detect the signal. Electrons following resonant absorption originate either via the internal conversion or follow from the subsequent emission of the Auger electrons and eventually further emission of some various secondary electrons. The appearance of electrons following single absorption event is bunched on the time scale, of course. The Lorentzian approximation is justified due to the very short range of electrons in comparison with the absorption range of the resonant photon beam [1-3]. However, highly enriched targets cannot be described in the thin target limit. Hence, one has to take into account perturbations of the line shape due to the secondary (and eventually higher order) fields $[4,5]$. Therefore additional contribution to the line shape emerges and it resembles similar effects observed by using resonant detectors in the transmission geometry [3, 6-8] except dispersion terms, the latter being absent in the transmission geometry for the low energy M1 transitions. Some part of the secondary (and higher order) resonant field is coherent with the original field coming from the external source [9-11]. The presence of such contribution is the basis of the $\gamma$-ray holography in single crystals [12-15]. The effect is much smaller in the polycrystalline targets, but not absent entirely provided targets are highly enriched and eventually magnetized. The interference between primary and co-

\footnotetext{
* corresponding author; e-mail: sfrueben@cyf-kr.edu.pl
}

herent secondary fields leads to the line asymmetry [15]. The aim of the present contribution is to study highly enriched target, as such targets are essential to perform successfully $\gamma$-ray holography. On the other hand, one has to understand properly line shape modifications due to the coherent secondary field in order to obtain proper holographic reconstruction of the atomic positions.

The paper is organized as follows: Section 2 is devoted to the discussion of the secondary resonant radiation field, Section 3 deals with the evaluation of the (CEMS) and related spectra by suitable and realistic parameterization, and finally Section 4 is devoted to the evaluation of the Conversion Electron Mössbauer (CEM) spectrum recorded for the thick and highly enriched $\alpha$-Fe foil. The last section summarizes important conclusions.

\section{Secondary resonant radiation field in the CEM spectra}

Secondary (and eventually higher order) radiation field in the scattering experiment is due to the absorption of the primary field (single photon) and subsequent re-emission of the single photon. Some part of the secondary field is due to the elastic (coherent) Rayleigh scattering of the primary resonant field by the electrons - mainly atomic cores. A secondary photon could be absorbed again provided it has been emitted/scattered without recoil. The primary field is due to a photon emitted without recoil during transition between specified excited state of the nucleus and the ground state of this nucleus. The latter process occurs in the external source. Usually, one can neglect higher order fields than secondary as the intensity of the subsequent fields is getting rapidly smaller due to the electron conversion and emission/scattering with recoil. The secondary field adds to the primary field in either coherent or incoherent 
way. A coherent contribution comes from decays leading to the same state as the original ground state of the resonant nucleus, while the incoherent contribution is due to decays leading to different ground state than the original state. A coherent contribution is also due to the Rayleigh recoilless scattering by electrons.

Transition amplitude for the resonant absorption of the single photon, from the ground nuclear state $\left|\lambda_{\mathrm{g}}\right\rangle$ to the excited nuclear state $\left|\lambda_{\mathrm{e}}\right\rangle$, could be described by the following matrix element provided both of the above states are described by the semi-classical hyperfine Hamiltonians [16]:

$$
\begin{aligned}
& \left\langle\lambda_{\mathrm{e}}|\boldsymbol{T}(\boldsymbol{q}, \boldsymbol{\varepsilon})| \lambda_{\mathrm{g}}\right\rangle=\sqrt{p\left(\lambda_{\mathrm{g}}\right) f(\boldsymbol{q})} \sum_{L k} \sum_{m_{\mathrm{e}} m_{\mathrm{g}}}\left\langle\lambda_{\mathrm{e}} \mid I_{\mathrm{e}} m_{\mathrm{e}}\right\rangle \\
& \quad \times\left\langle I_{\mathrm{e}} m_{\mathrm{e}}\left|\boldsymbol{T}_{M k}^{(L p)}(\boldsymbol{q}, \boldsymbol{\varepsilon})\right| I_{\mathrm{g}} m_{\mathrm{g}}\right\rangle\left\langle I_{\mathrm{g}} m_{\mathrm{g}} \mid \lambda_{\mathrm{g}}\right\rangle .
\end{aligned}
$$

Symbols $\lambda_{\mathrm{g}}$ and $\lambda_{\mathrm{e}}$ denote respective eigenvalues of the hyperfine Hamiltonians, the symbol $\boldsymbol{q}$ stands for the wave-vector transfer to the system, while $\varepsilon$ denotes unit vector perpendicular to the wave-vector transfer and describing polarization of the absorbed single photon. A function $p\left(\lambda_{\mathrm{g}}\right)$ stands for the initial occupation of the $\left|\lambda_{\mathrm{g}}\right\rangle$ state (it is assumed that practically all nuclei remain in the ground state). Except for the lowest temperatures one can assume that the following condition is satisfied $p\left(\lambda_{\mathrm{g}}\right)=\left(2 I_{\mathrm{g}}+1\right)^{-1}$. The function $f(\boldsymbol{q})$ stands for the recoilless fraction dependent on the wave-vector transfer to the system. The symbol $L \geq 1$ stands for the angular momentum of the absorbed radiation, while the index $k= \pm 1$ enumerates two polarization states of radiation. Symbols $I_{\mathrm{g}}$ and $I_{\mathrm{e}}$ denote nuclear spins in the ground and excited state, respectively, while $m_{\mathrm{g}}$ and $m_{\mathrm{e}}$ stand for the respective nuclear magnetic quantum numbers. The symbol $p$ denotes nuclear transition parity, and finally $M=m_{\mathrm{e}}-m_{\mathrm{g}}$ stands for the magnetic quantum number of the absorbed photon, while the symbol $\boldsymbol{T}$ denotes multi-pole transition operator. Eigenvectors of the respective hyperfine Hamiltonians satisfy the following orthogonality condition:

$$
\sum_{m}\langle\lambda \mid \operatorname{Im}\rangle\left\langle\operatorname{Im} \mid \lambda^{\prime}\right\rangle=\delta_{\lambda \lambda^{\prime}} .
$$

The symbol $\delta_{\lambda \lambda^{\prime}}$ stands for the Kronecker symbol. Multi-pole operator satisfies standard selection rules, i.e., $M=0,1, \ldots, L$ and $\left|I_{\mathrm{e}}-I_{\mathrm{g}}\right| \leq L \leq I_{\mathrm{e}}+I_{\mathrm{g}}$. Respective nuclear magnetic quantum numbers satisfy condition $-I \leq m \leq I$. The amplitude for nuclear resonant absorption takes on the form [13]:

$$
\begin{aligned}
& A(\omega \mid \boldsymbol{q}, \boldsymbol{\varepsilon})=\frac{\Gamma}{2} \sum_{\lambda_{\mathrm{e}} \lambda_{\mathrm{g}}}\left\langle\lambda_{\mathrm{e}}|\boldsymbol{T}(\boldsymbol{q}, \boldsymbol{\varepsilon})| \lambda_{\mathrm{g}}\right\rangle \\
& \times \frac{\Gamma / 2-\mathrm{i}\left[\omega-\left(\lambda_{\mathrm{e}}-\lambda_{\mathrm{g}}\right)\right]}{(\Gamma / 2)^{2}+\left[\omega-\left(\lambda_{\mathrm{e}}-\lambda_{\mathrm{g}}\right)\right]^{2}} \\
& =\sum_{\lambda_{\mathrm{e}} \lambda_{\mathrm{g}}} A\left(\omega\left|\lambda_{\mathrm{e}}, \lambda_{\mathrm{g}}\right| \boldsymbol{q}, \boldsymbol{\varepsilon}\right) .
\end{aligned}
$$

Here the symbol $\Gamma$ stands for the half-width of the resonant line, while $\omega$ denotes ambient energy.
Excited nucleus could decay into the ground state via emission of the recoilless single photon. The amplitude for such process is expressed as follows:

$$
\begin{aligned}
& \left\langle\lambda_{\mathrm{g}}^{\prime}\left|\boldsymbol{T}\left(\boldsymbol{q}^{\prime}, \boldsymbol{\varepsilon}^{\prime}\right)\right| \lambda_{\mathrm{e}}\right\rangle=\sqrt{\frac{b f\left(\boldsymbol{q}^{\prime}\right)}{1+\alpha}} \sum_{L^{\prime} k^{\prime}} \sum_{m_{\mathrm{e}}^{\prime} m_{\mathrm{g}}^{\prime}}\left[\left\langle\lambda_{\mathrm{g}}^{\prime} \mid I_{\mathrm{g}} m_{\mathrm{g}}^{\prime}\right\rangle\right. \\
& \left.\quad \times\left\langle I_{\mathrm{g}} m_{\mathrm{g}}^{\prime}\left|\boldsymbol{T}_{M^{\prime} k^{\prime}}^{\left(L^{\prime} p\right)}\left(\boldsymbol{q}^{\prime}, \boldsymbol{\varepsilon}^{\prime}\right)\right| I_{\mathrm{e}} m_{\mathrm{e}}^{\prime}\right\rangle\left\langle I_{\mathrm{e}} m_{\mathrm{e}}^{\prime} \mid \lambda_{\mathrm{e}}\right\rangle\right] .
\end{aligned}
$$

The symbol $b$ stands for the branching ratio leading from the excited state to the respective ground nuclear state (for the first excited state $b=1$ ), while $\alpha$ denotes total conversion coefficient for the nuclear transition involved. Total amplitude for nuclear excitation and decay to the resonant radiative state can be expressed as $\left\langle\lambda_{\mathrm{g}}^{\prime}\left|\boldsymbol{T}\left(\boldsymbol{q}^{\prime}, \boldsymbol{\varepsilon}^{\prime}\right)\right| \lambda_{\mathrm{e}}\right\rangle\left\langle\lambda_{\mathrm{e}}|\boldsymbol{T}(\boldsymbol{q}, \boldsymbol{\varepsilon})| \lambda_{\mathrm{g}}\right\rangle$. The process is coherent provided the following condition is satisfied $\lambda_{\mathrm{g}}^{\prime}=\lambda_{\mathrm{g}}$. Otherwise re-radiated field is incoherent with the original excitation electromagnetic field.

Let us assume that the sample is illuminated by the primary resonant radiation coming from the unpolarized single line and resonantly thin source. One can assume that primary field is due to single decay in the source [13]. Amplitude of such radiation at the average position of the absorbing nucleus has the following form for distant source:

$$
\begin{aligned}
& A_{0}(\omega-v|z| \boldsymbol{q}, \boldsymbol{\varepsilon})=\sqrt{\frac{\Gamma}{2 \pi}}\left(\frac{\Gamma / 2-\mathrm{i}(\omega-v)}{(\Gamma / 2)^{2}+(\omega-v)^{2}}\right) \\
& \quad \times \exp \left(-\frac{N \sigma}{2}[A(\omega \mid \boldsymbol{q}, \boldsymbol{\varepsilon})+(A+\mathrm{i} B)] z\right) \\
& \quad \times \exp \left(-\frac{\mu z}{2}\right) .
\end{aligned}
$$

Here the symbol $v$ stands for the first order Doppler shift applied along the primary beam (all spectral shifts are taken with respect to the source of primary resonant radiation). The symbol $N$ stands for the density of the resonant nuclei within the target and $\sigma$ stands for the nuclear cross-section for the resonant absorption. Symbols $A$ and $B$ stand for the real and imaginary part of the amplitude (divided by $\sqrt{\sigma}$ ) responsible for the recoilless Rayleigh scattering by the electrons (here mainly core electrons of the atoms - the majority of them being resonant). Above amplitude has very small admixture of the nuclear Thomson and Delbrück amplitudes. The symbol $z$ denotes length of the path traveled by the primary beam within the target prior to being absorbed. A decrement $\mu$ describes resonant beam attenuation due to the incoherent non-resonant processes. Let us assume that all resonant atoms are equivalent to each other and that they fill completely regular positions of the Bravais lattice, the latter lattice having definite orientation with respect to the incoming primary resonant beam. It is assumed that other scattering centers than the resonant atoms could be neglected. Coherent excitation amplitude of the nucleus located on the average at the origin is due to the primary field and coherent part of the secondary field. It could be expressed as follows: 


$$
\begin{aligned}
& A_{\mathrm{Coh}}(\omega-v|z| \boldsymbol{q}, \boldsymbol{\varepsilon})=\sqrt{\sigma} A_{0}(\omega-v|z| \boldsymbol{q}, \boldsymbol{\varepsilon}) A(\omega \mid \boldsymbol{q}, \boldsymbol{\varepsilon}) \\
& +\sqrt{\sigma} \sum_{n} A_{0}\left(\omega-v\left|z_{n}\right| \boldsymbol{q}, \boldsymbol{\varepsilon}\right)\left[A\left(\omega \mid-\boldsymbol{q}_{n}, \boldsymbol{\varepsilon}_{n}\right)\right. \\
& +A+\mathrm{i} B] \sum_{\lambda_{\mathrm{e}} \lambda_{\mathrm{g}}}\left\langle\lambda_{\mathrm{g}}\left|\boldsymbol{T}\left(\boldsymbol{q}_{n}, \boldsymbol{\varepsilon}_{n}\right)\right| \lambda_{\mathrm{e}}\right\rangle A\left(\omega\left|\lambda_{\mathrm{e}}, \lambda_{\mathrm{g}}\right| \boldsymbol{q}, \boldsymbol{\varepsilon}\right) \\
& \quad \times \exp \left(-\frac{1}{2} N \sigma\left[A\left(\omega \mid-\boldsymbol{q}_{n}, \boldsymbol{\varepsilon}_{n}\right)\right.\right. \\
& \left.+(A+\mathrm{i} B)] \sqrt{\boldsymbol{R}_{n} \cdot \boldsymbol{R}_{n}}\right) \\
& \times \frac{\exp \left(\mathrm{i}\left[\left(\boldsymbol{q}_{n}-\boldsymbol{q}\right) \cdot \boldsymbol{R}_{n}+q\left(z_{n}-z\right)\right]\right)}{\sqrt{(4 \pi) \boldsymbol{R}_{n} \cdot \boldsymbol{R}_{n}}} \\
& \times \exp \left(-\frac{1}{2} \mu \sqrt{\boldsymbol{R}_{n} \cdot \boldsymbol{R}_{n}}\right) \quad \text { with } q=|\boldsymbol{q}| .
\end{aligned}
$$

The summation goes over all lattice sites $n$ except origin. The symbol $\boldsymbol{R}_{n}$ denotes position of the average particular lattice site with respect to the (relative) origin, while $\boldsymbol{q}_{n}$ is the wave-vector of the secondary resonant radiation being co-axial with $\boldsymbol{R}_{n}$. The first term is responsible for the excitation by the primary field, while the second term is responsible for the excitation by the secondary coherent field including coherent elastic field due to the Rayleigh scattering. The latter field is generated by all resonant nuclei in the target except the nucleus at the origin and by distant electrons. One has to note that the amplitude for the Rayleigh scattering depends on $\boldsymbol{q}-\boldsymbol{q}_{n}$ and polarization of the incoming and outgoing radiation.

The incoherent excitation amplitude due to some distant resonant nucleus could be expressed as follows for the same wave-vector transfer and polarization as for the coherent excitation amplitude (it is assumed that elastic incoherent Rayleigh field is almost negligible):

$$
\begin{aligned}
& A_{\text {Inc }}\left(\omega-v\left|n, z_{n}, \lambda_{\mathrm{g}}, \lambda_{\mathrm{g}}^{\prime}\right| \boldsymbol{q}, \boldsymbol{\varepsilon}\right) \\
& =\sqrt{\sigma} A_{0}\left(\omega-v\left|z_{n}\right| \boldsymbol{q}, \boldsymbol{\varepsilon}\right) A\left(\omega \mid-\boldsymbol{q}_{n}, \boldsymbol{\varepsilon}_{n}\right) \\
& \times \frac{\exp \left(-\frac{1}{2} N \sigma\left[A\left(\omega^{\prime} \mid-\boldsymbol{q}_{n}, \boldsymbol{\varepsilon}_{n}\right)+(A+\mathrm{i} B)\right] \sqrt{\boldsymbol{R}_{n} \cdot \boldsymbol{R}_{n}}\right)}{\sqrt{(4 \pi) \boldsymbol{R}_{n} \cdot \boldsymbol{R}_{n}}} \\
& \quad \times \exp \left(-\frac{1}{2} \mu \sqrt{\boldsymbol{R}_{n} \cdot \boldsymbol{R}_{n}}\right)\left(1-\delta_{\lambda_{\mathrm{g}} \lambda_{\mathrm{g}}^{\prime}}\right) \\
& \quad \times \sum_{\lambda_{\mathrm{e}}}\left\langle\lambda_{\mathrm{g}}^{\prime}\left|\boldsymbol{T}\left(\boldsymbol{q}_{n}, \boldsymbol{\varepsilon}_{n}\right)\right| \lambda_{\mathrm{e}}\right\rangle A\left(\omega\left|\lambda_{\mathrm{e}}, \lambda_{\mathrm{g}}\right| \boldsymbol{q}, \boldsymbol{\varepsilon}\right),
\end{aligned}
$$

with $\omega^{\prime}=\omega+\left(\lambda_{\mathrm{g}}-\lambda_{\mathrm{g}}^{\prime}\right)$ and $\lambda_{\mathrm{g}} \neq \lambda_{\mathrm{g}}^{\prime}$.

Finally, excited nucleus decays to some of the ground hyperfine states via emission of the conversion electron (either without or with recoil). The conversion process occurs for one of the available electronic states of the resonant atom. This process is partly coherent with the emission of the photoelectron from the same electronic state.

Total cross-section for the emission of the electron by the resonant atom due to particular polarization and wave-vector transfer from the incoherent field is obtained as:

$$
\begin{aligned}
& S_{\mathrm{Inc}}(\omega-v|z| \boldsymbol{q}, \boldsymbol{\varepsilon})=\sum_{n} \sum_{\lambda_{\mathrm{g}} \lambda_{\mathrm{g}}^{\prime}} \\
& \times\left(A_{\mathrm{Inc}}^{*}\left(\omega-v\left|n, z_{n}, \lambda_{\mathrm{g}}, \lambda_{\mathrm{g}}^{\prime}\right| \boldsymbol{q}, \boldsymbol{\varepsilon}\right) F^{*}\right)\left(\begin{array}{cc}
1 & \beta_{0} \\
\beta_{0}^{*} & 0
\end{array}\right) \\
& \times \quad\left(\begin{array}{c}
A_{\mathrm{Inc}}\left(\omega-v\left|n, z_{n}, \lambda_{\mathrm{g}}, \lambda_{\mathrm{g}}^{\prime}\right| \boldsymbol{q}, \boldsymbol{\varepsilon}\right) \\
F
\end{array}\right) .
\end{aligned}
$$

Here the symbol $F$ denotes complex amplitude for the emission of the photoelectron, while the symbol $\beta_{0}$ denotes projection parameter of the amplitude $F$ on the nuclear amplitude with $\beta_{0} \beta_{0}^{*} \leq 1$. Corresponding cross-section due to the coherent field takes on the form:

$$
\begin{gathered}
S_{\mathrm{Coh}}(\omega-v|z| \boldsymbol{q}, \boldsymbol{\varepsilon})=\left(A_{\mathrm{Coh}}^{*}(\omega-v|z| \boldsymbol{q}, \boldsymbol{\varepsilon}) F^{*}\right) \\
\quad \times\left(\begin{array}{cc}
1 & \beta_{0} \\
\beta_{0}^{*} & 0
\end{array}\right)\left(\begin{array}{c}
A_{\mathrm{Coh}}(\omega-v|z| \boldsymbol{q}, \boldsymbol{\varepsilon}) \\
F
\end{array}\right) .
\end{gathered}
$$

Complete cross-section describing emission of the electron due to the above processes (coherent and incoherent) is expressed as proportional to:

$$
\begin{aligned}
& S(\omega-v|z| \boldsymbol{q}, \boldsymbol{\varepsilon})=S_{\mathrm{Coh}}(\omega-v|z| \boldsymbol{q}, \boldsymbol{\varepsilon}) \\
& \quad+S_{\mathrm{Inc}}(\omega-v|z| \boldsymbol{q}, \boldsymbol{\varepsilon}) .
\end{aligned}
$$

One has to note that the amplitude $F$ might depend on the particular ground hyperfine-state in the magnetically ordered target [17-19] due to the spin polarization of the s-like electron shells of the resonant atom. However this effect is negligible provided all conversion electrons are counted instead of the electrons emerging from the outer shells. The latter partial coherency has local character, as it occurs within the atomic shell of the resonant atom. This local coherence is particularly strong for E1 nuclear transitions as photo-effect and Rayleigh scattering occurs mainly via the E1 interaction for low energy photons.

The last expression has to be averaged over all polarization states of the photons involved and integrated over the ambient energy and photon paths. One has to introduce probability for the electron emergence from the target as well. Hence, one obtains finally:

$$
S(v, \boldsymbol{q})=\int_{-\infty}^{+\infty} \mathrm{d} \omega \int \mathrm{d} z P(z)\langle S(\omega-v|z| \boldsymbol{q}, \boldsymbol{\varepsilon})\rangle_{\left\{\boldsymbol{\varepsilon}_{n}\right\}, \boldsymbol{\varepsilon}} .
$$

Here the function $P(z)$ stands for the probability density function for the electron generated at the $\operatorname{depth} z$ to reach the detector and to be registered. The bracket $\langle\ldots\rangle$ denotes averaging operation.

Total signal from the detector takes on the following form provided detector behaves linearly and the source motion amplitude is small compared to the source-target distance:

$$
P(v, \boldsymbol{q})=B_{0}+A_{0}[S(v, \boldsymbol{q}) / \sigma] .
$$

Here the parameter $B_{0}$ stands for the number of counts due to detector background, while $A_{0}$ denotes overall 
phenomenological spectrum amplitude. The above formalism could be easily extended for multiple sites of the resonant atom and/or to the higher than secondary order fields $[13,15]$. For any significant kind of disorder within the target on the mesoscopic scale one has to perform further averaging of the Eq. (12), i.e.:

$$
P(v)=(4 \pi)^{-1} \int_{0}^{2 \pi} \mathrm{d} \phi \int_{0}^{\pi} \mathrm{d} \theta \sin \theta P(\theta, \phi) P(v, \boldsymbol{q})
$$

with $\boldsymbol{q}=\sqrt{\boldsymbol{q} \cdot \boldsymbol{q}}\left(\begin{array}{c}\sin \theta \cos \phi \\ \sin \theta \sin \phi \\ \cos \theta\end{array}\right)$. Here the function $P(\theta, \phi)$ denotes probability density function for a particular orientation of the crystal (including magnetic structure) versus incoming beam. Angles $\theta$ and $\phi$ denote polar and azimuthal angle, respectively in the chosen uniquely co-ordinate system. Usually integration of the Eq. (13) has to involve some partial averaging over the ensemble $\left\{\boldsymbol{R}_{n}, \boldsymbol{q}_{n}\right\}$ as the secondary field could propagate from one to another crystallite (domain).

Equation (12) is the basis of the $\gamma$-ray holography with the external reference wave and internal detector [12-15]. Basically, the useful information about atomic positions is contained in the line shape. This shape varies with the wave-vector transfer to the system, i.e., with the varying beam orientation versus single crystal axes.

\section{Evaluation of the CEM spectra originating in the highly enriched target}

It is obvious that above formalism is too complex to be directly applicable to process real spectra. Such situation is due to the very complex character of the secondary resonant field propagation in the highly enriched targets. Fortunately one can simplify above general description for particular situations. Things are getting simpler for almost completely resolved hyperfine lines, pure nuclear transitions connecting ground state with the first excited state and for diagonal hyperfine Hamiltonians with completely lifted degeneracy. In particular for the low energy nuclear M1 transition amplitudes for the Rayleigh scattering and photoelectron emission are almost orthogonal to the nuclear emission/absorption amplitude. Hence, one can set $\beta_{0}=0$ without introducing errors. One can neglect as well interference between decay into radiative channel and Rayleigh scattering amplitudes on the resonant atom being final detector. Hence, one has Lorentzian shape for absorption in the transmission geometry in such cases [20-22].

Let us consider almost monochromatic radiation beam, the latter beam having negligible divergence and negligible polarization. Let the beam falls on the flat and macroscopically homogeneous target, the latter target having very large thickness and being composed of the more or less randomly oriented crystallites on the macroscopic scale. It is assumed that the beam axis makes the right angle with the target surface. Hence, the signal from the secondary radiation detector $P(v)$ due to the secondary radiation emerging from the target could be approximated as follows provided the detector remains in the linear regime and the distance between the source of radiation and the target remains almost constant at any time:

$$
\begin{aligned}
& P(v)=B_{0}+S_{0} N \int_{-\infty}^{+\infty} \mathrm{d} \omega \rho(\omega-v)\left[\sigma_{\mathrm{R}} T(\omega)+\sigma_{0}\right] \\
& \quad \times \int_{0}^{+\infty} \mathrm{d} z p(z) \exp \left(-N\left[\sigma_{\mathrm{R}} T(\omega)+\sigma_{0}\right] z\right) .
\end{aligned}
$$

The symbol $S_{0}$ stands for the amplitude of the useful signal, while the function $\rho(\omega-v)$ denotes density of the resonant radiation from the source versus frequency $\omega$ and applied first order Doppler shift $v$ along the beam axis. The symbol $\sigma_{\mathrm{R}}$ denotes effective cross-section for the resonant absorption of the photon by the nucleus i.e. it depends on the recoilless fraction for the incoming beam. The symbol $\sigma_{0}$ stands for the cross-section for the non-resonant absorption, and $T(\omega)$ denotes resonant absorption profile within the target and versus frequency $\omega$, i.e., $T(\omega)$ describes dependence of the absorption on the ambient energy. A condition $|T(\omega)| \leq 1$ is always satisfied. The function $p(z)$ denotes probability of the secondary radiation to emerge from the target versus depth from the target surface, the latter depth being denoted by $z$. It is convenient to use dimensionless function $p(z)$ instead of previously described function $P(z)$. Remaining symbols have the same meaning as in the previous section. For single line source characterized by the negligible resonant self-absorption one has the following radiation density in accordance with Eq. (5):

$$
\rho(\omega-v)=\left(\frac{\Gamma_{s}}{2 \pi}\right)\left(\frac{1}{\left(\Gamma_{s} / 2\right)^{2}+(\omega-v)^{2}}\right),
$$

where $\int_{-\infty}^{+\infty} \mathrm{d} \omega \rho(\omega-v)=1$ and the symbol $\Gamma_{s}$ stands for the half-width of the emitted resonant line. For many cases probability for the secondary non-resonant radiation emergence from the target takes on the form $p(z)=\exp \left(-N \sigma_{\mathrm{A}} z\right)$. Here the symbol $\sigma_{\mathrm{A}}$ denotes effective cross-section for the secondary radiation absorption in the target. The above function is quite accurate for secondary photons having well defined energy and approximate for secondary electrons of any kind [3, 23]. Under such circumstances Eq. (14) could be transformed to the following form upon having performed integration over target thickness:

$$
\begin{gathered}
P(v)=B_{0}+\left(\frac{S_{0} \sigma_{0}}{\sigma_{0}+\sigma_{\mathrm{A}}}\right)+A \int_{-\infty}^{+\infty} \mathrm{d} \omega \rho(\omega-v) \\
\times \frac{T(\omega)}{1+\beta T(\omega)}=B_{0}+\left(\frac{S_{0} \sigma_{0}}{\sigma_{0}+\sigma_{\mathrm{A}}}\right)+A\left(\frac{\Gamma_{s}}{2 \pi}\right) \\
\quad \times \int_{-\infty}^{+\infty} \mathrm{d} \omega \frac{1}{\left(\Gamma_{s} / 2\right)^{2}+(\omega-v)^{2}} \frac{T(\omega)}{1+\beta T(\omega)} .
\end{gathered}
$$

Phenomenological parameter $\beta>0$ (for $\beta=0$ one obtains exact thin target approximation) is defined as $\beta=\sigma_{\mathrm{R}} /\left(\sigma_{0}+\sigma_{\mathrm{A}}\right)>0$. One obtains reasonable thin target approximation for $\beta \ll 1$. Another phenomenological 
parameter is defined as $A=S_{0} \beta$. Hence, the number of counts per data channel far off the resonance amounts to: $C_{0}=B_{0}+S_{0} \sigma_{0} /\left(\sigma_{0}+\sigma_{\mathrm{A}}\right)$.

One has to note that Eq. (16) allows for non-Lorentzian primary radiation densities as well $[24,25]$. Actually single line spectra remain Lorentzian, albeit the line broadens with the increasing parameter $\beta$. For multiple line spectra relative intensities depend on $\beta$, i.e., they tend to equalize for large values of $\beta$.

For highly enriched sample one can expect significant secondary resonant radiation field $[4,5]$. Usually one can neglect other radiation than resonantly scattered radiation and elastic Rayleigh scattered radiation for such situation [13]. Under such circumstances the function $T(\omega)$ could be expressed as follows:

$$
\begin{aligned}
& T(\omega)=N_{0}^{-1} \sum_{\kappa=1}^{\mathrm{K}} C_{\kappa}\left(\frac{\Gamma_{0}}{\Gamma_{\kappa}\left(1+\delta_{\kappa}\right)}\right) \\
& \times\left[\frac{\left(\Gamma_{\kappa} / 2\right)^{2}}{\left(\Gamma_{\kappa} / 2\right)^{2}+\left(\omega-\omega_{\kappa}\right)^{2}}\left(1-\left(\frac{2 \psi_{\kappa}}{\Gamma_{\kappa}}\right)\left(\omega-\omega_{\kappa}\right)\right)\right. \\
& \quad+\delta_{\kappa}\left(\frac{\left(\Gamma_{\kappa} / 2\right)^{2}}{\left(\Gamma_{\kappa} / 2\right)^{2}+\left(\omega-\omega_{\kappa}\right)^{2}}\right)^{2} \\
& \left.\quad \times\left(1-\left(\frac{2 \varphi_{\kappa}}{\Gamma_{\kappa}}\right)\left(\omega-\omega_{\kappa}\right)\right)\right]
\end{aligned}
$$

with $N_{0}=\sum_{\kappa=1}^{\mathrm{K}} C_{\kappa}$. The summation goes over all lines. The parameter $\Gamma_{0}$ stands for the natural half width of the transition, the symbol $\Gamma_{\kappa}$ denotes half-width of the particular line, while the symbol $\omega_{\kappa}$ stands for the position of this line. Relative intensities of subsequent lines are described by parameters $C_{\kappa} \geq 0$. Secondary field (originating by the nuclear scattering) contributing to the particular line is described by the parameter $\delta_{\kappa}$. It is assumed that all local interference terms (on the resonant atom undergoing final decay by emission of the conversion electron) due to the Rayleigh scattering amplitude and emission of the photoelectron are absent. A contribution due to the coherent part of the secondary field is accounted for in the first order approximation by introduction of the term depending on the parameter $\varphi_{\kappa}$. The latter parameter could vary from line to line as various lines have different contributions due to the coherent secondary field. Similar parameters $\psi_{\kappa}$ account for the interference of the primary field with the coherent part of the secondary field on the nucleus undergoing subsequent decay via emission of the conversion electron. Namely, terms $\psi_{\kappa}$ are responsible for the interference of the primary resonant field with the elastic Rayleigh field due to the distant electrons, while terms $\varphi_{\kappa}$ are responsible for the interference between coherent secondary resonant field (of the nuclear origin) and above Rayleigh field. These terms are present due to the fact that elastic Rayleigh field coming from distant electrons is able to excite resonant nucleus.

\subsection{Case of metallic iron}

A resonant transition we are interested in occurs in ${ }^{57} \mathrm{Fe}$ between the ground state and first excited state lying $14.41 \mathrm{keV}$ above the ground state of this stable nucleus. It is practically pure M1 transition between $I_{\mathrm{g}}=\frac{1}{2}^{(-)}$and $I_{\mathrm{e}}=\frac{3}{2}^{(-)}$nuclear states. Metallic iron has BCC structure at room temperature and it is very soft ferromagnetic material with the easy axis of magnetization aligned with one of the edges of the chemical unit cell. Hence, the hyperfine splitting is due to the pure magnetic dipole interaction between nuclear magnetic moment and the unique hyperfine magnetic field on the iron nucleus. Line positions depend on the magnetic coupling in the ground state, on the magnetic coupling in the excited state, and total shift versus applied single line (unpolarized) source. Magnetic splitting is sufficient to resolve almost completely particular lines. All absorption lines are Lorentzian in shape, and therefore Eq. (17) applies here. The line pattern is shown in Fig. 1. For completely random orientation of the magnetic domains one obtains the following ratio of the subsequent line intensities $3: 2: 1: 1: 2: 3$ as the recoilless fraction is practically isotropic here. However iron foils tend to acquire some preferential magnetization due to the shape anisotropy. They are usually partly magnetized in the foil plane, i.e., perpendicular to the incoming beam of radiation. Some additional in-plane magnetization might occur due to the surface anisotropy. The latter contribution is seen in the CEM spectra, as the signal comes from the relatively thin layer located close to the sample surface. This effect could be accounted for by introduction of the $g_{11}^{111}$ element of the reduced irreducible tensor $g_{M M^{\prime}}^{L_{0} L L^{\prime}}$. The latter element is real number and the previously introduced intensities transform into the following set of corrected intensities $3 g_{11}^{111}: 2: g_{11}^{111}: g_{11}^{111}: 2: 3 g_{11}^{111}$. Elements $g_{M M^{\prime}}^{L_{0} L L^{\prime}}$ are defined as $g_{M M^{\prime}}^{L_{0} L L^{\prime}}=\alpha_{M M^{\prime}}^{L L^{\prime}} / \alpha_{00}^{L_{0} L_{0}}$, where $L_{0}=\min \{L\}$ and the following relationship holds $[26,27]$ :

$$
\begin{aligned}
& \alpha_{M M^{\prime}}^{L L^{\prime}}=\frac{\sqrt{(2 L+1)\left(2 L^{\prime}+1\right)}}{8 \pi} \\
& \quad \times \int_{0}^{2 \pi} \mathrm{d} \phi \exp \left(\mathrm{i}\left(M-M^{\prime}\right) \phi\right) \int_{0}^{\pi} \mathrm{d} \theta \sin \theta f(\theta, \phi) \\
& \quad \times \sum_{k= \pm 1}(k)^{L+L^{\prime}} d_{k M}^{L}(\theta) d_{k M^{\prime}}^{L^{\prime}}(\theta)
\end{aligned}
$$

with $f(\theta, \phi) \geq 0$ and $\frac{1}{4 \pi} \int_{0}^{2 \pi} \mathrm{d} \phi \int_{0}^{\pi} \mathrm{d} \theta \sin \theta f(\theta, \phi)=1$. The symbol $d_{k M}^{L}(\theta)$ stands for the matrix element of the generalized spherical harmonic, while the function $f(\theta, \phi)$ describes distribution of the magnetization axes with respect to the incoming beam. The element $g_{11}^{111}$ satisfies the following condition $g_{11}^{111}>\frac{1}{2}$. For foils magnetized perpendicular to the beam one has $g_{11}^{111}<1$. For isotropic distribution $f(\theta, \phi)$ one has $g_{11}^{111}=1$.

In principle all lines should have the same width in a transmission mode. However high precision transmission data exhibit usually slightly broader external lines $(1,6)$ 
in comparison with the pair $(2,5)$, and the latter pair is often slightly broader than the innermost pair $(3,4)$. This effect is due to the finite primary beam divergence. There is no observable difference between lines belonging to the same pair as the total shift of the spectrum is small compared to the splitting for available sources. Here the effect of the beam divergence is present as well. One can expect as well significant line broadening due to the fact that the final decay via emission of the conversion electron occurs from delocalized excited nuclear state due to the partly coherent nature of the excitation. Hence, the line widths could vary from one to another line.

One has to note that absorption due to one of the outermost lines does not lead to the incoherent secondary field upon subsequent decay with the emission of the recoilless photon. On the other hand, absorption due to either second or fifth line generates $1 / 3$ incoherent contribution to the secondary field. For innermost lines this contribution amounts to $2 / 3$. The angular distribution of the secondary field is different, while emitting lines $(2,5)$ in comparison with emission of the remaining lines. The overall intensity of the nuclear secondary field does not depend on the particular excitation transition.

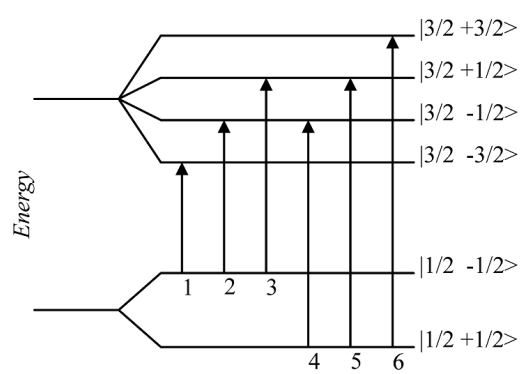

Fig. 1. Schematic representation of the absorption line pattern in metallic iron.

Hence, one can expect some further corrections to the line intensities due to the secondary field. Unfortunately, any corrections to the pair $(2,5)$ are masked by the much stronger effect of the partial magnetization. However, one can compare outermost pair with the innermost pair introducing the following set of intensities $3 g_{11}^{111}(1-\chi)$ : $2: g_{11}^{111}: g_{11}^{111}: 2: 3 g_{11}^{111}(1-\chi)$, where the parameter $\chi$ is adjustable.

\section{Experimental results and data evaluation}

A commercial resonantly thin and single Lorentzian line (with negligible polarization) ${ }^{57} \mathrm{Co}(\mathrm{Rh})-14.41 \mathrm{keV}$ source was used at room temperature under negligible pressure and without any external magnetic field. A target was made of thick polycrystalline $\alpha$-Fe foil enriched to 96 at.\% in ${ }^{57} \mathrm{Fe}$ and kept under the same thermodynamic conditions as the source. The low background gas flow CEMS detector was used in the back scattering geometry. A gas mixture was composed of helium with addition of 10 vol. $\% \mathrm{CH}_{4}$. The gas pressure was kept slightly above ambient pressure. Such detector has practically no energy resolution, hence it works in the integral CEMS mode, i.e., in the ICEMS mode. The lower threshold level was set in such way to remove detector noise, while the higher level threshold was set to discriminate against rare high-energy events. The spectrum was recorded in the round-corner triangular mode of the MsAa-3 spectrometer with the source being moved [28]. A dwell time for the raw data channel has been chosen as about $42.7 \mu \mathrm{s}$, while the number of raw data channels was selected as 4096. The folded and calibrated spectrum is shown in Fig. 2.

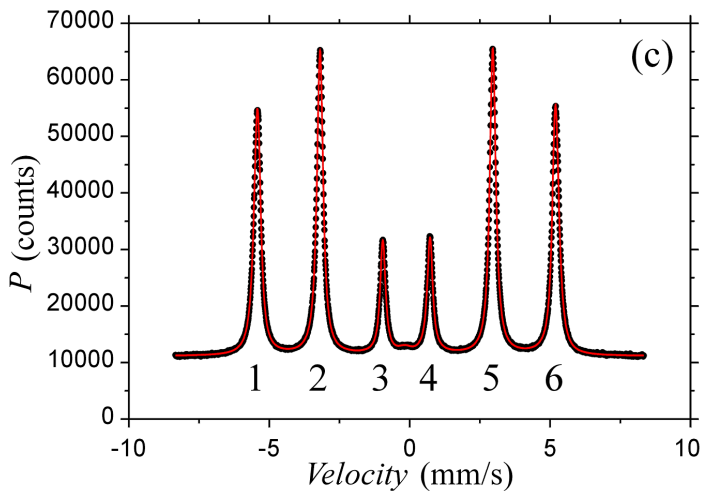

Fig. 2. Spectrum of the iron foil. Superimposed curve is obtained by fitting data within the final model (c). Iron lines are numbered in accordance with Fig. 1.

One can see slight oxidation of the iron foil surface as an additional broad feature close to the spectrum center. This imperfection was accounted for adding extra symmetric doublet composed of Lorentzian lines in each fit and described by four variables: two positions on the velocity scale, intensity and width. Such doublets are typical for iron foils exposed to the air and they are seen in the transmission spectra as well. The oxidation is only partial, and hence oxidized layer is highly disordered. Usually, it is composed of some iron hydroxides. The fitting procedure is based on Eqs. (16) and (17).

Data were fitted firstly within the thin target approximation and by using Lorentzian line-shapes model (a). The source line width was fixed to the natural line width $\Gamma_{s}=0.097 \mathrm{~mm} / \mathrm{s}$ and this parameter was kept constant at the above value in all subsequent fits as well. Parameters $\left\{\delta_{\kappa}, \varphi_{\kappa}, \psi_{\kappa}\right\}$ were set to zero in this model together with the parameter $\beta$. Remaining parameters were allowed to vary. Namely, the background, spectrum amplitude, magnetic coupling constants in the ground and excited state, total shift, line widths, $g_{11}^{111}$ and $\chi$ were allowed to vary. Oxide parameters were found in the separate run.

The second model (b) was extended in comparison with the model (a) allowing for additional variation of parameters $\left\{\delta_{\kappa}, \varphi_{\kappa}, \psi_{\kappa}\right\}$. Oxide parameters were kept constant on the previously determined values. Finally, the sensitivity of the spectrum to the variation of the parame- 


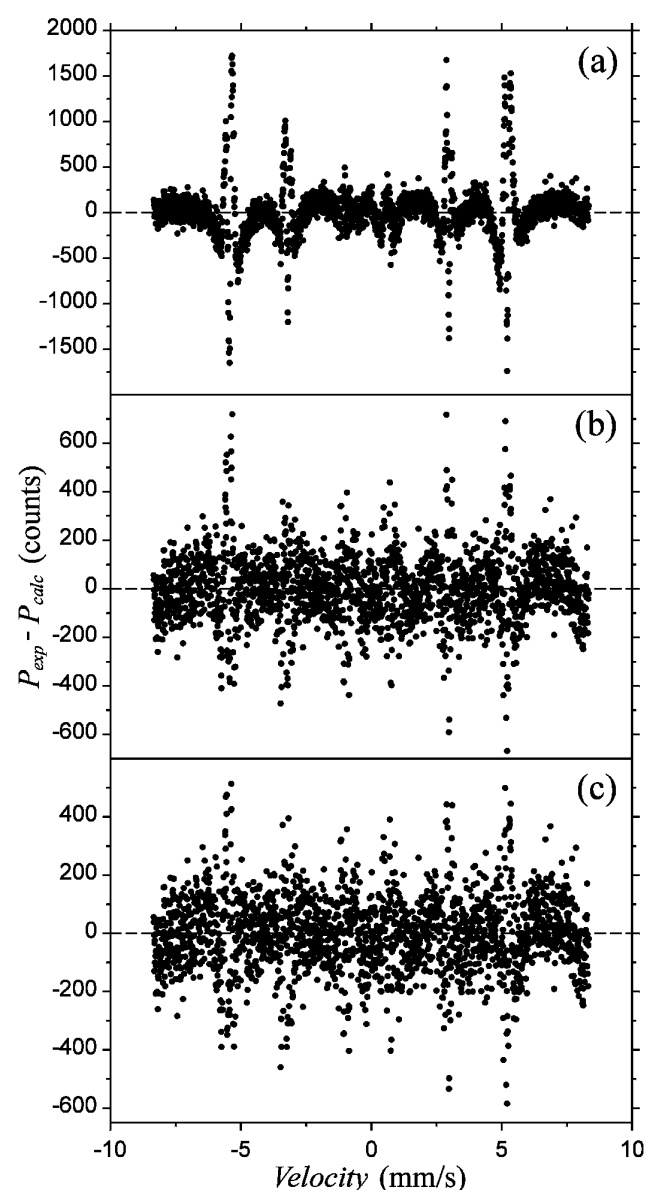

Fig. 3. Differential plots of residua for subsequent fitting models (a), (b), and (c) are shown versus velocity.

ter $\beta$ was investigated extending previous model (b) into final model (c). Oxide parameters were kept constant as above, and they were relaxed upon having received decent values of the remaining parameters. They were fixed in the final run on the corrected values. Figure 3 shows differential plots for final runs of above models.

It is clear that model (a) is unable to account for quite significant dispersion (interference) terms due to the secondary field. A transition to the model (b) accounts for the most of the line distortion due to the secondary field. Subsequent introduction of the target thickness in the model (c) has only minor effect as the cross-section for the low energy electrons scattering is extremely large in metallic iron. Some small deviations remain even within model (c) due to the unaccounted for higher than secondary fields and due to the histogram effect present even for the large number of data channels used (2047 for the folded spectrum). One can see some high order base line curvature as well, as the base line was treated within the single parameter approximation, as the major contributions to the curvature are removed by the folding procedure.
Figure 4 shows differential plots on the expanded velocity scale around strong line (2) for models (a) and (c) - final runs. A similar plot for completely "coherent" line (1) is shown in Fig. 5.

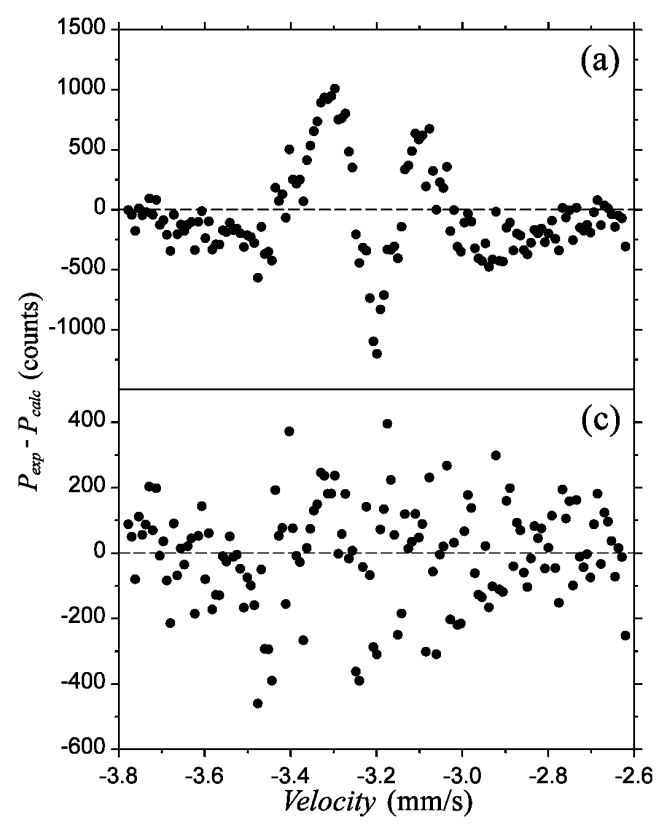

Fig. 4. Differential plots on the expanded velocity scale around strong line (2) for models (a) and (c).

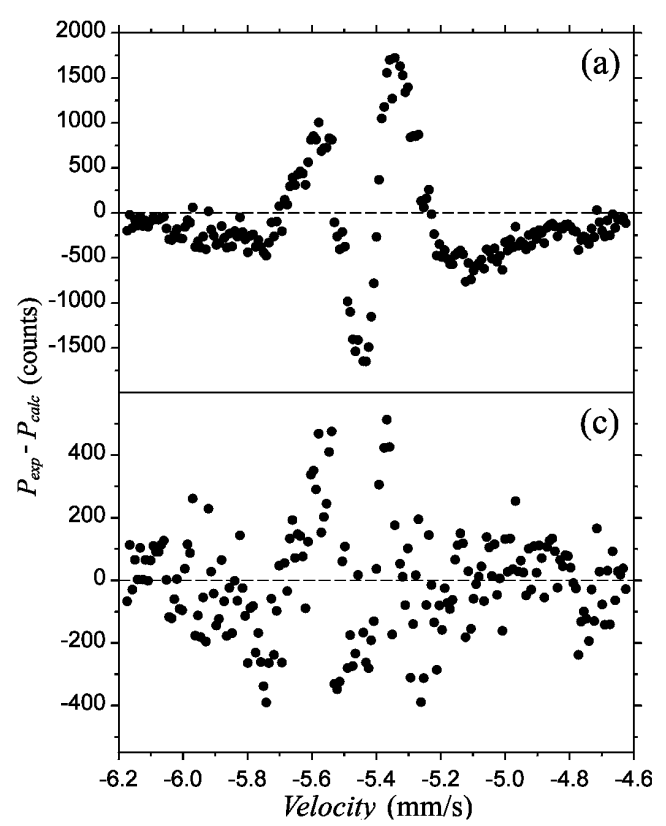

Fig. 5. Differential plots on the expanded velocity scale around completely "coherent" line (1) for models (a) and (c). 


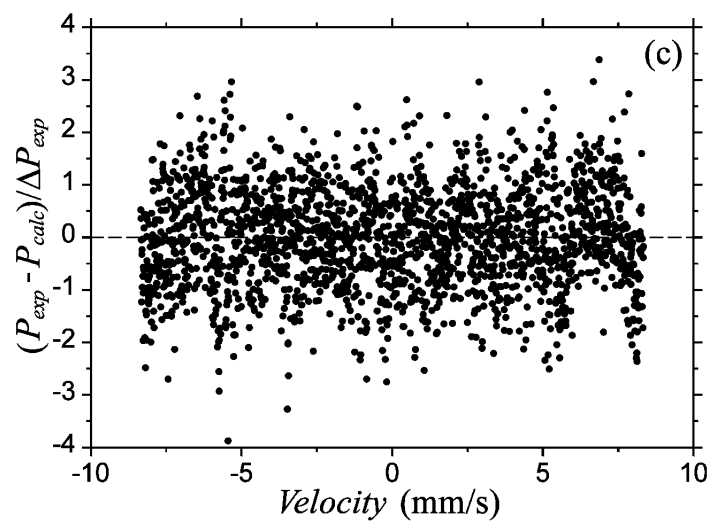

Fig. 6. Normalized differential plot for model (c). The symbol $\Delta P_{\exp }$ stands for the statistical error in each channel.

Complex dispersion term is clearly visible as the residuum of the model (a). This pattern is likely to vary for single crystal, while changing direction of the primary beam within the main crystal axes. Finally, the Table summarizes essential final parameters of the secondary field obtained within model (c). These parameters are to be treated as example as they can vary with the orientation of crystallites and overall target magnetization. It has to be noted that target line widths are quite broad beyond the geometrical (beam divergence) effect (see Table). It is strong indication for presence of the coherent excited nuclear state. Such state decays much faster than isolated nuclear state as it has more decay channels opened. Hence, it leads to the broadening of the line. Such state involves coherent recoilless radiation coupling various nuclei, and hence it depends on the degree of coherence during decay to the ground state. Thus external lines are broader than other lines, as they do not experience spin incoherence (see, Fig. 1) — they lead solely to the original ground hyperfine states. One has to realize that secondary radiation fields have much larger range than conversion electrons within target.

TABLE

Final parameters of the secondary field

\begin{tabular}{c|c|c|c|c}
\hline \hline Line $(\kappa)$ & $\delta_{\kappa}$ & $\varphi_{\kappa}$ & $\psi_{\kappa}$ & $\Gamma_{\kappa}[\mathrm{mm} / \mathrm{s}]$ \\
\hline 1 & $2.53(6)$ & $0.39(2)$ & $-0.027(2)$ & $0.246(1)$ \\
2 & $1.62(4)$ & $0.16(2)$ & $-0.007(1)$ & $0.185(1)$ \\
3 & $1.57(10)$ & $-0.45(5)$ & $0.050(5)$ & $0.178(2)$ \\
4 & $2.01(14)$ & $0.49(4)$ & $-0.090(6)$ & $0.179(2)$ \\
5 & $1.61(4)$ & $-0.11(2)$ & $-0.005(1)$ & $0.181(1)$ \\
6 & $2.35(6)$ & $-0.51(2)$ & $0.042(2)$ & $0.239(1)$
\end{tabular}

Parameters describing secondary field effects obtained within model (c) - final run. Line widths have been included as they are broadened due to the existence of the coherent excited nuclear state. The parameter $\chi$ has been obtained within model (c) as $\chi=-0.060(9)$, while the parameter $g_{11}^{111}$ has been obtained within the same model as $0.548(4)$.

Further development of the models is unjustified as one obtains $\chi^{2} /$ d.f. $=0.94$ and MISFIT $=0.0022(8) \%[29]$ in the case of model (c) - final run. The normalized differential plot for this model is shown in Fig. 6. Remaining structure does not justify further expansion of the model.

\section{Conclusions}

The secondary field modifies line shape, while the coherent part of this field leads to the modification of the line shape and to the broadening of lines as the coherent excited nuclear state is formed. The line shape is distorted by the interference terms (dispersion terms) due to the presence of the coherent secondary field generated by the elastic Rayleigh scattering of the primary radiation. Generally departures from the Lorentzian shape are small, however they are observable thanks to the very large signal to noise ratio for strongly enriched target. The thickness effect is small even for this strongly enriched target, as all conversion electrons have very small range in the metallic iron for this particular resonant nuclear transition. Above effects are practically undetectable for targets with natural isotopic composition of iron or only partly enriched. One has to note that a lot of coherence (dispersion terms) in the secondary field is lost for natural isotopic abundance of iron due to the isotopic incoherence, as resonant atoms are distributed almost randomly on the accessible iron sites.

\section{References}

[1] J.G. Mullen, J. Stevenson, Nucl. Instrum. Methods 153, 77 (1978).

[2] J.R. Gancedo, M. Garcia, J.F. Marco, Hyperfine Interact. 66, 83 (1991).

[3] A. Błachowski, K. Ruebenbauer, Phys. Scr. 73, 263 (2006).

[4] M. Tegze, G. Faigel, Europhys. Lett. 16, 41 (1991).

[5] M. Tegze, G. Faigel, Nature (London) 380, 49 (1996).

[6] K.P. Mitrofanov, N.V. Illarionova, V.C. Shpinel, Prib. Tekh. Eksp. 3, 49 (1963).

[7] J.G. Mullen, A. Djedid, C. Holmes, G. Schupp, M.L. Crow, W.B. Yelon, Nucl. Instrum. Methods B 14, 323 (1986).

[8] A.A. Belyaev, S.M. Irkayev, V.V. Panchuck, V.G. Semenov, V.S. Volodin, in: Proceedings of MSMS-2008, Eds. M. Mashlan, R. Zbořil, AIP Conference Proceedings, New York 2008, p. 147.

[9] P.I. Black, P.B. Moon, Nature (London) 188, 481 (1960).

[10] G.V. Smirnov, Hyperfine Interact. 97/98, 551 (1996).

[11] R. Rüffer, A.I. Chumakov, Hyperfine Interact. 97/98, 589 (1996).

[12] P. Korecki, J. Korecki, T. Ślęzak, Phys. Rev. Lett. 79 3518 (1997). 
[13] P. Korecki, J. Korecki, W. Karaś, Phys. Rev. B 59, 6139 (1999).

[14] P. Korecki, G. Materlink, J. Korecki, Phys. Rev. Lett. 86, 1534 (2001).

[15] P. Korecki, M. Szymoński, J. Korecki, T. Ślęzak, Phys. Rev. Lett. 92, 205501 (2004).

[16] M. Kwater, K. Ruebenbauer, U.D. Wdowik, Physica B 190, 209 (1993).

[17] C.J. Song, J. Trooster, N. Benczer-Koller, Phys. Rev. Lett. 29, 1165 (1972).

[18] C.J. Song, J. Trooster, N. Benczer-Koller, Phys. Rev. B 9, 3854 (1974).

[19] K. Burin, A.A. Manalio, J. Porellada, M.R. Polcari, G.M. Rothberg, J. de Physique 40, C2-188 (1979).

[20] W. Henning, G. Baehre, P. Kienle, Phys. Lett. B 31, 203 (1970).

[21] F.E. Wagner, B.D. Dunlap, G.M. Kalvius, H. Schaller, R. Felscher, H. Spieler, Phys. Rev. Lett. 28, 530 (1972).

[22] H. Prosser, F.E. Wagner, G. Wortmann, G.M. Kalvius, R. Wäppling, Hyperfine Interact. 1, 25 (1975).
[23] W.E. Burcham, Nuclear Physics, Longmans, London 1965.

[24] J.G. Mullen, A. Djedid, G. Schupp, D. Cowan, Y. Cao, M.L. Crow, W.B. Yelon, Phys. Rev. B 37, 3226 (1988).

[25] R. Wagoner, B. Bullard, M. May, S. Dickson, J.G. Mullen, Hyperfine Interact. 58, 2687 (1990).

[26] K. Ruebenbauer, Physica B 172, 346 (1991).

[27] J. Żukrowski, A. Błachowski, K. Ruebenbauer, J. Przewoźnik, D. Sitko, N.-T.H. Kim-Ngan, Z. Tarnawski, A.V. Andreev, J. Appl. Phys. 103, 123910 (2008).

[28] R. Górnicki, A. Błachowski, K. Ruebenbauer, Nukleonika 52, S7 (2007).

[29] S.L. Ruby, Mössbauer Effect Meth. 8, 263 (1973). 